\title{
Scar-Free Embryonic Wound Healing and the Prevention of Scarring Following Wound Healing in the Adult
}

\author{
R. L. MCCALLION and M. W. J. FERGUSON* \\ School of Biological Sciences, University of Manchester, 3.239 Stopford Building Manchester, M13 9PT, UK
}

\begin{abstract}
The development of scarring following accidental injury or surgery is an important clinical problem, often resulting in adverse effects on growth and function, as well as an undesirable cosmetic appearance. Adult wound healing and scar formation are characterised by acute inflammation, wound contraction and disordered collagen deposition. Similar processes may also be involved in the progression of fibrotic disease states such as pulmonary fibrosis and hepatic cirrhosis. A major clinical objective is therefore, the reduction, and ideally the prevention, of scarring. The embryo and early fetus respond to injury in a way that is fundamentally different from the adult. In general, the early fetus heals rapidly, without scar formation. Extracellular matrix deposition in the fetal wound is rapid and organised in its structure, much more similar in appearance to unwounded skin, whilst, in the adult, matrix deposition is disordered. The inflammatory response, characteristic of the early phase of adult wound healing, is absent, or highly limited, in the fetus and the levels of cytokines are generally greatly reduced. Research into fundamental cellular and molecular differences between adult and fetal wound healing have revealed a number of targets in the adult wound which can be manipulated to more closely resemble the fetal wound environment and hence result in the reduction of adult scarring.
\end{abstract}

Keywords: Fetal wound healing; adult wound healing; scarring; inflammation; extracellular matrix

\section{INTRODUCTION}

A scar can be defined as the macroscopic disturbance of normal skin structure and function, arising a consequence of wound repair (Ferguson et al., 1996). These macroscopic changes may be due to disruptions to the epidermal, dermal and subcutaneous tissues. A large body of evidence shows that the embryo and early fetus heal cutaneous wounds with the absence of scar formation. A number of parameters are critically important in determining if a fetal wound will scar and to what degree. A variety of animal species have been used in fetal wound-healing studies and the species of animal is critical to the results obtained. Species which have been extensively

The research reported in this paper was presented at the year long workshop on Mathematics in Medicine held at the International Centre for Mathematical Sciences, Edinburgh during 1994-1995.

${ }^{*}$ Corresponding author. Tel: 0161275 6775. Fax: 0161275 5945. E-mail: rmccalli@fs2.scg.man.ac.uk. 
studied include the rat (Goss, 1977; Robinson and Goss, 1981; Roswell, 1984), rabbit (Adzick et al., 1985a; Krummel et al., 1987), opossum (Ferguson and Howarth, 1991; Armstrong and Ferguson, 1995) and mouse (Martin and Lewis, 1992; Mccluskey et al., 1993; Whitby and Ferguson, 1991a).

The gestational age of an animal is an important factor in determining if a fetal wound will scar. In general, early embryos do not scar, whilst fetuses in the third trimester of development will heal similarly to the adult, with scar formation. In the rat, the transition from the scar-free to the scarring phenotype occurs between days 18 and 19 of gestation (Ihara et al., 1990), in the mouse between days 16 and 17 (Whitby and Ferguson, 1991a) whilst, in the sheep, this transition occurs between days 100 to 120 (Longaker et al., 1990a). The change from scarfree healing to the scarring phenotype of the adult is gradual in primates and in the rhesus monkey Lorenz and colleagues (1993) described a 'transition wound' between 85 and 100 days' gestation, in which wounds healed with an absence of sebaceous glands and hair follicles, but with reticular dermal collagen architecture similar to unwounded dermis. Prior to 85 days' gestation, the fetal wounds completely regenerated normal dermal architecture, whilst after 100 days' gestation, adult scarring was observed. This gradual transition from the scarfree to scarring phenotype has also been described by Armstrong and Ferguson (1995) in a marsupial model of wound healing. These authors described microscopic changes in the wounded dermis of the opossum which became more pronounced as the age of the pouch young at the time of wounding increased. These microscopic changes reflected a transition from no visible scar, to the obvious scarring response of the adult animal. The majority of investigations into the effect of gestational age on fetal scarring have employed a defined dermal incisional wound. However, Horne and colleagues (1992) studied excisional wounds in fetal lambs and reported scarring at gestational ages prior to 100 days. These studies demonstrate the critical importance of the severity of the wound on the scarring phenotype, showing a correlation between the extent of tissue damage and the gestational age at which the wound must be inflicted to achieve scar-free healing.

The site of the wound is another important parameter in determining if a fetal wound will scar. An incisional dermal wound in sheep made prior to 100 days' gestation will not scar, whilst similar wounds made in the alimentary tract, diaphragm and peritoneum all heal with scar formation (Adzick et al., 1985b; Longaker et al. 1991a; Meuli et al., 1995). There are similar known site-specific variabilities in the degree of scarring in the human.

The factors which control fetal healing may be extrinsic to the animal, a direct result of fetal environment, or intrinsic to the nature of fetal tissue. A thorough understanding of the relative importance of the underlying factors, controlling the fetal scarfree phenotype is vital to the goal of manipulation of the adult wound to achieve healing in the absence of a scar.

\section{FETAL ENVIRONMENT}

One of the most obvious differences between adult and fetal wound healing is the environment in which the wound heals. The characteristic warm, sterile, fluid environment may play a role in the scar-free healing of the fetus. Fetal wounds heal without the formation of a fibrin clot and it is thought that the absence of a scab may be a result of the fluid environment (Somasundaram and Pathap, 1972). The absence of the fibrin clot in fetal wound healing may be due to a combination of an immature fetal coagulation cascade and fibrinolytic activity of amniotic fluid. It is not yet clear what role, if any, the fibrin clot may play in the adult scarring phenotype. The clot may act as a reservoir of growth factors important in scarring and the role of the fibrin clot in adult wound healing merits investigation.

The extracellular matrix of the normal fetal dermis and fetal wounds is rich in hyaluronic acid (HA), a glycosaminoglycan (GAG) found at high concentrations in regions of tissue proliferation, repair and regeneration. The reasons for this high level of $\mathrm{HA}$ in the fetal dermis have not yet 
been fully determined. Fetal serum is rich in an HA stimulating factor, reaching a peak at $40 \%$ of gestation time, which may control HA deposition (Longaker et al., 1989). Amniotic fluid also contains an $\mathrm{HA}$ stimulating factor, similar to that found in fetal serum, as well as a high concentration of HA itself (Longaker et al., 1990b). The presence of this factor in serum and amniotic fluid may facilitate the rapid healing of fetal wounds, since high levels of HA may provide a matrix which favours rapid cell migration. Human amniotic fluid has also been shown to inhibit the activities of hyaluronidase, elastase and cathespin B, whilst enhanding collagenase activity (Gao et al., 1994). These findings may, in part, explain the high levels of HA found in fetal skin and the increased collagenase activity may have a role to play in the superior collagen fibre organisation seen in fetal wound healing.

Ammiotic fluid has also been reported to have effects on fetal wound contraction. Excisional fetal rabbit wounds do not contract when exposed to amniotic fluid. (Somasundaram and Prathap, 1970), but when excluded from the amniotic fluid by application of a silastic patch, contraction occurred rapidly (Somasundaram and Prathap, 1972). Hallock and colleagues (1988) also reported that fetal rat wounds do not contract in the presence of amniotic fluid, contrary to the work of Ihara and Motobayashi (1992), who observed wound contraction in rat fetal wounds of 16 days' gestation. However, the latter study was performed in vitro and this may account for the reported discrepancies. Longaker and colleagues (1991b), demonstrated that fetal lamb wounds contract in utero and that exposure to amniotic fluid had no significant effect on the rate of this contraction. In vitro studies have shown that sheep amniotic fluid enhances the contraction of a fibroblast-populated collagen lattice (FPCL) (Burd et al., 1991), whilst 21-week human amniotic fluid can inhibit FPCL contraction (Wider et al., 1993). Hence, the effects of amniotic fluid on wound contraction are species-dependent and the speciesspecific composition of amniotic fluid may explain these different effects.
The most direct evidence that the fetal environment is not of great significance in scar-free healing comes from studies carried out on the pouch young of marsupials. Monodelphis domesticus, the Brazilian grey short-tailed opossum, is born at a very early developmental stage, equivalent to a 6 week gestation human fetus (Morykwas et al., 1991) and is, therefore, externally accessible for woundhealing studies. In marsupial pouch young, the immune system is poorly developed, as is the case for eutherian mammals, but the skin is well developed and keratinised, thus avoiding dehydration. The use of Monodelphis domesticus as a woundhealing model allows investigation of the intrinsic factors important in wound healing, whilst the fetus is exposed to an adult environment. Armstrong and Ferguson (1995) showed that up to pouch day 4, the opossum demonstrated typical scar-free healing features in the adult environment with fibrin clot formation and wound contraction, from day 4 to day 9 some pouch young scarred, whilst after pouch day 9, all animals healed in an adult, scar-forming manner.

A number of studies have utilised grafts of fetal skin in the adult environment and the converse, to investigate the contribution of the fetal environment to scar-free healing. Wounds in adult sheep skin grafts on early gestation fetal lambs healed with the formation of a scar, whilst fetal-fetal transplants did not scar (Longaker et al., 1994). These investigations demonstrated that the fully differentiated skin of the adult cannot be modulated to heal in a scarfree fashion simply by perfusion with fetal serum or exposure to amniotic fluid. Wounds in grafts of human fetal skin, placed in a cutaneous position on a nude mouse, scarred regardless of the fetal age, whilst grafts placed in a subcutaneous pocket retained a scar-free healing phenotype (Lorenz et al., 1992). These studies demonstrated that the nonscarring phenotype of the fetus is intrinsic to fetal skin, although the location of the graft is critical. A more rapid rate of tissue differentiation, when fetal skin is exposed to a cutaneous environment (Lane et al., 1989) is thought to be the reason why fetal skin of the same gestational age will heal in 
a scar-free fashion in a subcutaneous location, but will scar when transplanted cutaneously.

Current evidence suggests that the fetal environment is not an essential component of scar-free healing and that the degree of fetal differentiation is the most important determinant of scarring. Advancing tissue maturity, increasingly complex dermal structure, cellular differentiation and immune system maturity all correlate with the diminishing ability to heal without scarring with increasing gestational age.

\section{INFLAMMATION AND CYTOKINES}

In adult wound healing, one of the initial events is platelet degranulation resulting in the immediate release of a number of cytokines including plateletderived growth factor (PDGF), epidermal growth factor (EGF) and transforming growth factor-beta $(\mathrm{TGF} \beta)$. Neutrophils are immediately attracted to the wound site, where they phagocytose bacteria and damaged tissue. Macrophage infiltration follows and neutrophils are ingested by the invading macrophages. The influx of both neutrophils and macrophages is directly mediated by TGF $\beta$ (Wahl et al., 1987). The invading macrophages are activated by the cytokines released from the platelets and then produce a wide variety of cytokines themselves, including interleukin-1 (IL-1), basic fibroblast growth factor (bFGF), PDGF, TGF $\alpha$ and $\mathrm{TGF} \beta$, resulting in positive feedback for the recruitment of more macrophages. TGF $\beta$ and PDGF are also potent chemoattractants for fibroblasts and stimulate them to proliferate and produce new extracellular matrix constituents. These stimulated fibroblasts also produce TGF $\beta$ and PDGF in an autocrine feedback loop, similar to macrophages (Pierce et al., 1991). The factors which bring about resolution of the inflammatory response have not yet been thoroughly elucidated. The production of 'anti-inflammatory' cytokines, such as IL-10, which inhibits TGF $\beta$ synthesis (Van Vlasselaer et al., 1994) may be important in switching off the inflammatory response and the deposition of collagen may attenuate the response of dermal fibroblasts to TGF $\beta$ (Clark et al., 1995).

Fetal wound healing is fundamentally different from wound healing in the adult, since very little inflammation occurs during repair. A direct consequence of the lack of inflammatory response in the fetus may be the absence of scarring. Evidence directly implicates high levels of specific growth factors, particularly TGF $\beta_{1+2}$ with scarring and fibrosis. The fibrotic potential of TGF $\beta$ has been shown when levels remain elevated. Intravenous injections of TGF $\beta$ in the rat produced marked fibrosis of the liver and kidney and also at the site of injection (Terrell et al., 1993). The distribution of growth factors in fetal wounds was investigated by Whitby and Ferguson (1991b), who studied the immunolocalisation of PDGF, TGF $\beta$ and $\mathrm{bFGF}$ in fetal, neonatal and adult mouse wounds. PDGF was detected at the site of all wounds within 1 hour of wounding, but the rate of clearance from the different wound types varied with age. In the fetal wound, PDGF was not detected by 48 hours postwounding; in the neonate, staining disappeared at 72 hours post-wounding, at which time PDGF was still present in the adult. TGF $\beta_{1}$ and $\beta_{2}$ were not detected within the fetal wounds at any time points studied, but were observed at 1,6 and 12 hours postwounding at the wound surface and within the clot of both neonatal and adult wounds. Basic FGF was not detected within fetal wounds, but was observed in adult and neonatal wounds at 1 and 6 hours after wounding. The authors suggested that the persistence of PDGF within the adult wound may be due to the synthesis of this growth factor by cells such as macrophages which are recruited to the adult wound site but are absent from fetal wounds. The lack of TGF $\beta$ within the fetal wounds may also be due to the lack of infiltrating inflammatory cells, a lack of degranulating platelets upon wounding, or an absolute lack of TGF $\beta$ from fetal platelets. These authors also implicated the lack of inflammatory response in the fetus as a secondary cause of the absence of bFGF. The lack of staining for $\mathrm{TGF} \beta$ and bFGF reported by these authors does not indicate an absolute absence of these cytokines from fetal wounds 
but reduced levels below the detection limits of the immunocytochemical techniques employed.

The distribution of TGF $\beta_{1}$ in the wounds of fetal mice was also investigated by Martin and colleagues (1993), using in situ hybridisation. Transcripts for TGF $\beta_{1}$ synthesis were found to be rapidly induced in epithelial cells within 1 to 3 hours of wounding and by 3 to 6 hours in the wound bed. TGF $\beta_{1}$ protein was detected by immunocytochemistry within 1 hour of wounding and by 8 hours after wounding the levels of TGF $\beta_{1}$ staining had returned to background levels. This was in contrast to the level of growth factor in the adult wound, where sustained levels of TGF $\beta_{1}$ protein was detected (Kane et al., 1991). Hopkinson-Woolley and colleagues (1994) confirmed the lack of macrophage infiltration into the fetal wound, the cell type which is the most potent and sustained source of TGF $\beta_{1}$. Macrophage recruitment during fetal wound healing in the mouse was also investigated by these authors. Embryos were wounded at gestation times of 11.5 to 14.5 days and it was found that macrophage recruitment into the wound site did not occur until gestation day 14.5. The ability of macrophages from 11.5-day gestation mice to respond to chemotactic stimuli was studied by inflicting burn wounds to produce localised tissue necrosis. A significant number of macrophages were recruited to the wound site in response to the burn injury, illustrating that embryonic macrophages are capable of mounting an inflammatory response in the presence of an appropriate stimulus. Hence, the severity and type of wound is an important determinant in the inflammatory response elicited in the fetus and therefore the degree of scarring. A similar correlation between the ability to mount an inflammatory response and the onset of scarring was observed in the pouch young of the marsupial Monodelphis domesticus (Armstrong and Ferguson, 1995).

\section{EXTRACELLULAR MATRIX}

The regulation of cell function by the extracellular matrix (ECM) is a fundamental mechanism controlling cell phenotype and behaviour. The ECM is an important reservoir for growth factors and may interact with these molecules in an antagonistic or synergistic fashion. Interactions between the individual components of the ECM and specific cell-surface molecules can initiate a series of signal transduction events, resulting in varied cellular responses. Many studies have shown striking differences between the ECM of fetal and adult wounds, particularly, the restoration of normal tissue collagen architecture in scarless fetal wounds. Differences in the ECM profile between fetal and adult wounds may cause changes in the behaviour of infiltrating cells which may determine if a wound heals with a scarring or scar-free phenotype. The constituents of the ECM and the kinetics of their deposition may be important parameters influencing scarring.

Collagen is the major structural protein of scar tissue in post-natal animals and many studies have focused on collagen deposition in wounds using different species, wound models and ages of animal. Collagen is deposited in fetal wounds at a greater rate than in the adult and in a normal reticular pattern, rather than the scarred pattern of an adult wound. Burd and colleagues (1990) demonstrated quantitative differences in fetal and adult wound collagen content in sheep. Biochemical analysis of hydroxyproline content showed an increase in fetal and adult wounds over 15 days after wounding. Thereafter, adult wound collagen levels fell, whereas levels in fetal wounds (75-100 days' gestation) continued to rise. Franz and colleagues (1992) quantified the rate of collagen synthesis in fetal and adult rabbit wounds. In adults, there was a preferential stimulation of collagen synthesis compared to non-collagenous protein synthesis from 5 to 10 days post-wounding, but in the fetus, both collagen and non-collagenous protein synthesis were elevated for the first 5 days after wounding.

The authors attributed the delay of onset of collagen synthesis in the adult animals to a number of factors. Adult fibroblasts are in a quiescent state and relatively sparse throughout the normal dermis. They require time for proliferation and migration into the wound space before collagen synthesis 
begins. However, fetal fibroblasts are in a relatively active state and may require no specific stimulation to up-regulate collagen synthesis. Moreover, fetal fibroblasts are capable of proliferation and collagen synthesis simultaneously, whereas adult fibroblasts only produce significant amounts of collagen when not dividing (Graham et al., 1984). Whitby and Ferguson (1991a) carried out a detailed immunocytochemical study of the ECM deposited in fetal, neonatal and adult lip wounds in mice. These authors found that collagens types I, III, IV, $\mathrm{V}$ and VI were present in wounds from animals of all ages, but that the timing and pattern of collagen deposition varied with age. In the fetus and neonate, collagen was detected by 48 hours post-wounding, but did not appear in adult wounds until 5 days post-wounding. All five types of collagen examined appeared almost simultaneously. The major difference between collagens in the fetal and adult wounds was in the organisation of collagen fibrils. In the fetus, collagen was deposited in a reticular fashion, indistinguishable from the surrounding dermis, whereas, in the adult, the collagen fibrils were deposited in dense, parallel bundles, typical of scar tissue. This illustrates the critical difference in control and patterning of collagen fibrillogenesis between fetal and adult wounds. Collagen fibrillogenesis is, as yet, incompletely understood. It is known that types I, II and V are present within the same fibrils and that interactions between the different collagen types can alter fibril size in vitro (Birk et al., 1990). Proteoglycans can also alter collagen fibril size (Scott, 1988), as can fibronectin (Speranz et al., 1987). Therefore, collagen fibrillogenesis may be influenced by the existing ECM environment. It is well established, in a number of species, that fetal tissues contain a higher proportion of type III collagen compared to type I than found in the adult (Epstein, 1974; Merkel et al., 1988). The adult ratio of collagen types do not become established until 10-15 days' post-partum (Morykwas et al., 1991; Boon et al., 1992; Hallock et al., 1993). The ratio of type III to type I collagen may influence fibre size, but since fetal wounds heal with scarring, long before transition to the adult type III to type I collagen ratio, it is unlikely that these collagen ratios are of major significance in the scar-free phenotype.

Fibronectin (Fn) is expressed at high levels during wound healing, before the appearance of collagen and is a major component of the primary ECM during tissue repair. Fn is involved in the migration of all major cell types into the wound and may act as a provisional matrix for ECM assembly. Differences in the deposition of $\mathrm{Fn}$ in fetal wound healing have been investigated in full-thickness linear incisions made on adult rabbits and fetuses of 24 days' gestation (Longaker et al., 1989). Wounds were harvested between 4 and 24 hours' post-wounding and the total Fn was detected by immunocytochem-. istry. In the fetus, Fn was first detected 4 hours after wounding, becoming more prominent until the staining was very strong and covered the wound edge by 24 hours' post-wounding. In contrast, in the adult wound, Fn deposition was not detected until 12 hours' post-wounding. These authors suggested that the earlier deposition of $F n$ in the fetal wound may provide an earlier signal for cell migration and hence, underlie the rapid re-epithelialisation of the fetal wound compared to the adult. Whitby and Ferguson (1991a), showed that Fn was present at the surface of the wounds in adult, fetal and neonatal mice by 1 hour post-wounding. This staining persisted up to 72 hours in 16- and 18-day gestation fetal wounds, but was present up to 7 days in neonatal and adult wounds. The decrease in Fn staining observed was concomitant with increasing collagen deposition. Wounding of adult tissue has been shown to result in the reappearance of embryonic forms of spliced Fn (French-Constant et al., 1989), which may be more functionally appropriate in terms of wound repair. The normal existence of embryonic Fn splicing in the fetal wound may contribute to the accelerated healing observed, since there is no requirement for the type of Fn to be switched. It takes approximately 4 days for the embryonic type of Fn to appear in significant quantities in the adult wound (Shaw and Olsen, 1991) and the different isoforms of Fn present at the time of wounding may be important in influencing the rate of healing. 
The major glycosaminoglycans (GAGs) in skin are hyaluronic acid (HA), dermatan sulfate (DS) and chondrotin sulfate (CS), with a minor contribution from heparan sulfate (HS) (Mast et al., 1992). HA is the most extensively studied GAG in fetal and adult wound healing and as yet, little is known about the possible role of other GAGs and proteoglycans in wound healing. It has long been known that the ECM becomes enriched with HA coincident with episodes of rapid cellular migration and proliferation. HA is believed to stimulate cell proliferation and migration by creating a low-resistance hydrated matrix that removes cells from contact inhibition and restricted mobility (Brecht et al., 1986). HArich matrices are thought to bind growth factors, thus influencing cell growth and differentiation by changing the local concentration of these cytokines (Ruoslahti and Yamoguchi, 1991). It is also believed to regullate cell behaviour by the provision of ligands for cell attachment and motility (Turley et al., 1991). Fetal skin contains much higher levels of HA than adult skin. The ECM of fetal wounds is rich in HA compared to the transient up-regulation of HA at the adult wound site, a finding observed in both the rabbit (DePalma et al., 1989; Mast et al., 1991; Stern et al., 1992) and the sheep (Chiu et al., 1990; Longaker et al., 1991b). Estes and colleagues (1993) suggested that high and prolonged levels of HA in fetal wounds promoted scar-free healing and showed that a decrease in wound HA content, with increasing fetal age correlated with the onset of scarring in 120-day gestation lamb fetuses. HA concentration may also be an important factor in determining the inflammatory response at the wound site. Dillon and colleagues (1994) examined the adherence of fetal lymphocytes to a number of ECM components and found that these cells showed no binding to HA, the most abundant component of fetal ECM.

Fibroblasts are critical to the wound-healing process, since it is these cells which are responsible for the deposition and remodeling of most of the new ECM. After migration into the wound space, fibroblasts assume a synthetic phenotype and begin to synthesize collagen and Fn. Fetal fibroblasts can both proliferate and synthesize collagen simultaneously (Graham et al., 1984), in contrast to adult fibroblasts and this difference in phenotype may directly contribute to the faster rate of fetal healing. Schor and colleagues (1985) demonstrated that fetal fibroblasts will migrate into a collagen three-dimensional gel, whereas few normal adult fibroblasts will do so. These authors proposed that a novel protein, migration stimulation factor (MSF), produced by fetal fibroblasts, was the factor responsible for the migratory phenotype of the fetal cells. MSF may increase cell migration by stimulating their production of HA (Schor et al., 1989) and it has been suggested that MSF may be related to HASA detected in amniotic fluid and fetal serum (Longaker et al., 1990a). The restriction of fibroblast motility and low HA concentrations in the adult wound may directly contribute to the adult scarring phenotype, since the adult oral mucosa, which secretes high levels of MSF and has many fetal-like characteristics, does not scar (Irwin et al., 1994). The change in phenotype of fibroblasts in adult granulation tissue, with expression of $\alpha$ smooth muscle actin (ASMA), was first described by Gabbiani and colleagues (1972). These cells were termed 'myofibroblasts' and are also present permanently in fibrocontractive diseases, such as hepatic cirrhosis and pulmonary fibrosis (Schurch et al., 1992). The current prevailing hypothesis is that the myofibroblast is responsible for wound contraction. Estes and coworkers (1994) described the development and ultrastructural features of myofibroblasts in fetal lamb wounds from 75 to 120 days' gestation. ASMA staining was absent from normal skin and wounds of 75-day gestation animals, except in vascular smooth muscle cells and pericytes. However, by 100 days' gestation, substantial ASMA staining was observed in fetal wounds, increasing from 3 to 7 days' postwounding. ASMA staining was also strongly positive in the wounds of sheep of 120 days gestation. When the wound fibroblasts were cultured in vitro, all expressed ASMA, including those from 75-day gestation animals, indicating that the fibroblasts from early gestation fetuses have the capability of expressing ASMA, but lack the 
necessary signals to do so in vivo. The transition from scar-fiee to the scarring phenotype in the sheep coincides with the appearance of ASMA expression by wound fibroblasts and the lack of myofibroblasts in non-scarring fetuses may be due to the lack of an appropriate stimulus, such as TGF $\beta$. The causes and consequences of wound contraction are not yet fully clear, but it is possible that the mechanism of wound contraction has a direct bearing on the degree of scarring. Lorenz and colleagues (1995) have investigated the role of fetal fibroblasts in scar-free healing by transplanting human fetal fibroblasts into wounds on athymic mice. These authors showed that subcutaneous grafts healed with human fetal collagen and no scar formation, whereas cutaneous grafts healed with mouse collagen in a typical scarring pattern and suggested that the fetal fibroblast is the major effector cell for scar-free repair.

\section{MANIPULATION OF THE ADULT WOUND TO REDUCE SCARRING}

Research into the fundamental differences between adult wound healing and fetal scar-free healing have suggested several areas in which the adult wound could be manipulated to more closely resemble the fetus, in the hope of reducing scarring. The major areas in which attention has been focused are the modulation of the inflammatory response and growth factor profile within the adult wound and manipulation of the ECM components towards a more fetal-like profile. The most striking difference between the ECM of fetal and adult wounds is the high concentration of HA at the fetal wound site, hence a number of workers have attempted to reduce adult scarring by the application of exogenous HA to the wound site. Hellstrom and Laurent (1987) applied HA to the edges of rat tympanic perforations and found that the healing rate was accelerated from closure in an average of 12 days to healing in 7 days. Further studies indicated that HA application also markedly affected the structural quality of the healed membrane, with beneficial effects on scarring seen within 1 month (Laurent et al., 1988). More recently, Cabera and colleagues (1995) studied the effect of an HA-associated protein collagen complex on scarring in the rat. This group found a significant reduction in scarring when the complex was applied by intradermal injection to the margins of incisional wounds, or as a cream to excisions. However, pretreatment with hyaluronidase did not abrogate the effect of the complex, whilst protease digestion abolished the effect. It was therefore concluded that it was the, as yet unknown, protein associated with the complex which was responsible for the reduction in scarring observed, rather than the HA itself. In our laboratory, the addition of pure, fermentation produced HA to adult, incisional rat wounds was found to have no obvious effects on dermal scarring (McCallion et al., submitted).

The significance of TGF $\beta$ in scarring and fibrosis has been documented by many workers. Studies have been carried out in our laboratory to determine the contribution of $\operatorname{TGF} \beta$ to the scarring process. Neutralising antibody to $\mathrm{TGF} \beta_{1+2}$ was injected intradermally into adult rat wounds, just prior to wounding and on days 1 and 2 postwounding. A significant effect on scarring was observed by 42 days post-wounding when antibody treated wounds displayed much reduced scarring, with collagen fibre orientation much more similar to unwounded dermis than the dense, aligned collagen fibres seen in untreated scars (Shah et al., 1992). At 7 days post-wounding, neutralising antibodytreated wounds had a significantly reduced monocyte and macrophage profile, substantially reduced numbers of new blood vessels and less collagen and fibronectin within the wounds (Shah et al., 1994). The neutralising antibody must be administered at the time of wounding, or very soon thereafter, for significant anti-scarring effects to be observed. Reduction of the TGF $\beta_{1+2}$ levels immediately after wounding may prevent further amplification of this growth factor at the wound site. Application of neutralising antibodies to TGF $\beta$ have also proved effective in the reduction of fibrosis in conditions such as glomerulonephritis (Border et al., 1990) and pulmonary fibrosis (Giri et al., 
1993). Isoform specific antibodies were also applied to wounds, to determine the relative contribution of each isoform of TGF $\beta$ to the scarring response (Shah iet al., 1995). It was found, that for a significant anti-scarring effect both $\mathrm{TGF} \beta_{1}$ and TGF $\beta_{2}$ must be neutralised. Surprisingly, exogenous addition of TGF $\beta_{3}$, at low concentrations was also found to havie marked anti-scarring activity (Shah et al., 1995). This work clearly demonstrates isoform specific differences in the roles of TGF $\beta$ in wound healin $\&$ and scarring.

TGF $\beta$ s are secreted with a latency associated peptide (LAP), as part of a biologically inactive complex, unable to interact with cell-surface receptors. Two of the three oligosaccharide side chains of the LAP have mannose-6-phosphate (M6P) as their terminal residues (Purchio et al., 1988). M6P binds specifically and with high affinity to the cation independent M6P/insulin-like growth factor-II (IGFII) recieptor and binding of LAP to this receptor appears to be required for the activation of TGF $\beta$ (Dennis and Rifkin, 1991). Studies carried out in our laboratory, using rat and pig wounds, have shown that addition of M6P to the wound reduces scarring and has similar effects on the healing process as the addition of neutralising antibody to TGF $\beta_{1+2}$ (McCallion et al., submitted).

Collectively, these studies in which the adult wound is manipulated to more closely resemble healin $\$$ in the fetus, suggest that there is a large therapeutic window for improving the quality of adult wound repair. Such manipulations may involve alterations to the concentrations of a number of active growth factors, or manipulation of the ECM composition. Identification of the most important parameters will be facilitated by further study into the differences between adult and fetal wounds.

\section{References}

Adzick, N. S., Harrison, M. R., Glick, P. L., Beckstead, J. H. Villa, R. L., Schevevstuhl, H. and Goodson, W. H. (1985a) "Comparison of fetal, newborn and adult wound healing by histologic, enzyme-histochemical and hydroxyproline determination," J. Pediatric Surg., 20, 315-319.

Adzick, A. S., Outwater, K. M.. Harrison, M. R., Davies, P. Glick, P. L., deLorimer, A. A. and Reid, L. M. (1985b) "Correction of congenital, diaphragmatic hernia in utero. IV.
An early gestational fetal lamb model for pulmonary vascular morphometric analysis," J. Pediatric Surg., 20, 673-680.

Armstrong, J. R. and Ferguson, M. W. J. (1995) "Ontogeny of the skin and the transition from scar-free to scarring phenotype during wound healing in the pouch young of Monodelphis domestica," Develop. Biol., 169, 242-260.

Birk, D. E., Fitch, J. M., Barbiaz, J. P., Doane, K. J. and Linsenmayer, T. F. (1990) "Collagen fibrillogenesis in vitro: Interaction of types I and V collagen regulates fibril diameter," J. Cell Sci, 95, 649-657.

Border, W. A., Okuda, S., Languino, L. R., Sporn, M. B. and Ruoslahti, E. (1990) "Suppression of experimental glomerulonephritis by anti-serum against transforming growth factor$\beta, "$ Nature, 346, 371-374.

Boon, L., Manicourt, D., Marbaix, E., Vandenabeele, M. and Vanwijck, R. (1992) "A comparative analysis of surgical cleft lip corrected in utero and in neonates," Plastic Reconstruct. Surg., 89, 11-17.

Brecht, M., Mayer, U. and Schlosser, E. (1986) "Increased hyaluronic acid synthesis is required for fibroblast detachment and mitosis," Biochem. J., 239, 445-450.

Burd, D. A. R., Longaker, M. T., Adzick, N. S., Harrison, M. R. and Erlich, H. P. (1990) "Fetal wound healing in a large animal model: The deposition of collagen is confirmed," Brit. J. Plastic Surg., 43, 571-577.

Burd, D. A. R., Longaker, M. T., Rittenberg, T., Adzick, N. S., Harrison, M. R. and Erlich, H. P. (1991) "In vitro fetal wound contraction: the effect of amniotic fluid," Brit. J. Plastic Surg., 44, 302-305

Cabera, R. C., Siebert, J. W., Eidelman, Y., Gold, L. I., Longaker, M. D. and Garg, H. G. (1995) "The in vivo effect of hyaluronan associated protein-collagen complex on wound repair," Biochem. Molec. Biol. Int., 37, 151-158.

Chandrakasan, G., Rukta, J. and Stern, R. (1986) "Hyaluronic acid stimulates collagen synthesis and levels of type III collagen in cultures of human fibroblasts" (Abstract), J. Cell Biol, 103, 252.

Chiu, E. S., Longaker, M. T., Adzick, N. S., Stern, M., Harrison. M. P. and Stern, R. (1990) "Hyaluronic patterns in fetal and adult wound fluid," Surg. Forum, 41, 636-639.

Clark, R. A. F., Nielsen, L. D., Welch, M. P. and McPherson, J. M. (1995) "Collagen matrices attenuate the collagensynthetic response of cultured fibroblasts to TGF $\beta, " J$. Cell Sci., 108, 1251-1261.

Dennis, P. A. and Rifkin, D. B. (1991) "Cellular activation of latent TGF $\beta$ requires binding to the cation independent M6P/IGF II receptor," Proc. Natl. Acad. Sci. USA, 88, 580-584.

DePalma, R. L., Krummel, T. M., Durham, L. A., Michna, B. A., Thomas, B. L., Nelson, J. M. and Diegelmann, R. F. (1989) "Characterisation and quantitation of wound matrix in the fetal rabbit," Matrix, 9, 224-231.

Dillon, P. W., Keefer, K., Blackburn, J. H., Houghton, P. E. and Krummel, T. M. (1994) "The extracellular matrix of the fetal wound: Hyaluronic acid controls lymphocyte adhesion," $J$. Surg. Res., 57, 170-173.

Epstein, E. H. (1974) "(Alpha 1 (3)) human skin collagen. Release by pepsin digestion and preponderance in fetal life," J. Biol. Chem., 249, 3225-3231.

Estes, J. M., Adzick, N. S., Harrison, M. R., Longaker, M. T. and Stern, R. (1993) "Hyaluronate metabolism undergoes an ontogenic transition during fetal development: Implications for scar-free wound healing," J. Pediatric Surg.. 28, 1227-1231.

Estes, J. M., Van de Berg, J. S., Adzick, N. S.. MacGillivray, T. E., Desmouliere, A. and Gabbiani, G. (1994) "Phenotypic 
and functional features of myofibroblasts in sheep wounds," Differentiation. 56. 173-181.

Ferguson, M. W. J. and Howarth, G. F. (1991) "Marsupial models of scarless fetal wound healing." In Fetal Wound Healing, N. S. Adzick and M. T. Longaker, eds, pp. 92-125. Elsevier, Amsterdam

Ferguson, M. W. J., Shah, M., Armstrong, J., Whitby. D. J. and Longaker, M. T. (1996) "Scar formation. The spectral nature of fetal and adult wound repair," Plastic Reconstruct. Surg., 97, $854-859$.

French-Constant, C., Van De Water, L.. Dvorak, H. F. and Hynes, R. O. (1989) "Reappearance of an embryonic pattern of fibronectin splicing during wound healing," J. Cell Biol., 109, $903-914$.

Franz, F. W., Dieglmann, R. F., Mast, B. A. and Cohen, K. (1992) "Biology of fetal wound healing: Collagen biosynthesis during dermal repair," J. Pediatric Surg., 27, 945-949.

Gabbiani, G., Hirschel, B. J., Ryan, G. B., Statkov, P. R. and Majno, G. (1972) "Granulation tissue as a contractile organ: A study of structure and function," J. Experimental Med., 135 . $719-734$.

Gao, X. X., Devoe, L. D. and Given, K. S. (1994) "Effects of amniotic fluid on proteases - A possible role of amniotic fluid in fetal wound healing," Ann. Plastic Surg., 33. 128-134.

Giri, S. N., Hyde, D. M. and Hollinger, M. A. (1993) "Effect of antibody to transforming growth factor- $\beta$ on bleomycin induced accumulation of lung collagen in mice." Thorax, 48. $959-966$.

Goss, A. N. (1977) "Intrauterine healing of fetal rat oral mucosal, skin and cartilage wounds." J. Oral Pathol. 6. 35-38.

Graham, M. F., Diegelmann, R. F. and Cohen, I. K. (1984) "An in vitro model of fibroplasia: Simultaneous quantification of fibroblast proliferation, migration and collagen synthesis." Proc. Royal Soc. Experimental Med., 176, 302-308.

Hallock, G., Merkel, J. R., Rice, D. C. and Dipaolo, B. R. (1993) "The ontogenic transition of collagen deposition in ral skin," Ann. Plastic Surg., 30, 310-315.

Hallock, G., Rice, D. C., Merkel, J. R. and DiPaolo, B. R. (1988) "Analysis of collagen content in the fetal wound," Ann. Plastic Surg., 21, 310-315.

Hellstrom, S. and Laurent, C. (1987) "Hyaluronan and healing of tympanic membrane perforations: An experimental study," Acta Otolaryngol., S442, 54-61

Hopkinson-Woolley.

Horne, R. S. C.. Hurley, J. V., Crowe, D. M.. Ritz, M. H. McCo'Brien, B. and Arnold. L. I. (1992) "Wound healing in fetal sheep: A histological and electron microscope study," Brit. J. Plastic Surg., 45, 333-345.

Ihara, S. and Motobayashi, Y. (1992) "Wound closure in fetal rat skin," Development, 114, 573-582.

Ihara, S., Motobayashi, Y., Nagao, E. and Kistler, A. (1990) "Ontogenic transition of wound healing pattern in rat skin occurring at the rat fetal stage." Development. 110, 671-680.

Irwin, C. R., Picardo, M.. Ellis. I.. Sloan, P.. Grey. A. M. McGurk, M. and Schor, S. L. (1994) "Inter-and intra-site heterogeneity in the expression of fetal-like phenotypic characteristics by gingival fibroblasts: Potential significance for wound healing," J. Cell Sci., 197, 1333-1346

Kane, C. J. M., Mansbridge, J. N., Hebda. P. A. and Hanawalt, P. C. (1991) "Direct evidence for spatial and temporal regulation of transforming growth factor $\beta_{1}$ expression during cutaneous wound healing," J. Cell Phwsiol., 148, 157-173.

Krummel, T. M., Nelson, J. M., Diegelmann. R. F., Linblad W. J., Salzberg, A. M., Greenfield, L. J. and Cohen, I. K. (1987) "Fetal response to injury in the rabbit," J. Pediatric Surg., 22, 640-644.
Lane, A. T., Scott, G. A. and Day. K. H. (1989) "Development of fetal skin transplanted onto nude mice." $J$. Investigative Dermatol., 93, 787-791.

Laurent. T. C.. Hellstrom, S. and Fellenius, E. (1988) "Hyaluronan improves the healing of experimental membrane perforations," Arch. Otolaringol., S442, 7-24.

Longaker, M. T., Harrison, M. R.. Crombleholme, T. M., Langer, J. C.. Decker. M., Verrier, E. D., Spendlove, R, and Stern, R. (1989) "Studies in fetal wound healing: I. A factor in fetal serum that stimulates deposition of hyaluronic acid," J. Pediatric Surg.. 24. 789-792.

Longaker, M. T., Whitby, D. J., Adzick. N. S.. Crombleholme, T. M.. Langer, J. C., Duncan, B. W., Bradley, S. M., Stern, R.. Ferguson, M. W. J. and Harrison. M. R. (1990a) "Studies in fetal wound healing. VI. Second and early third trimester fetal wounds demonstrate rapid collagen deposition without scar formation," J. Pediatric Surg., 25, 63-68.

Longaker, M. T., Adzick, A. S., Hall, J. L., Stair, S. E., Crombleholme, T. M., Duncan, B. W., Bradley, S. M., Harrison. M. R. and Stern. R. (1990b) "Studies in fetal wound healing: VII. Fetal wound healing may be modulated by hyaluronic acid stimulating activity in amniotic fluid," $J$. Pediatric Surg., 25, 430-433.

Longaker, M. T.. Whitby, D. J., Jennings, R. W., Duncan, B. W., Ferguson, M. W., Harrison. M. R. and Adzick, N. S. (1991a) "Fetal diaphragmatic wounds heal with scar formation," $J$. Surg. Res., 50, 375-385.

Longaker. M. T., Burd, A. R.. Gowen. A. H., Yen, T. S. B., Jennings, R. W.. Duncan. B. W., Harrison, M. R. and Adzick, N. S. (1991b) "Midgestational excisional fetal lamb wound contract in utero," J. Pediatric Surg., 26, 942-948.

Longaker, M. T., Whitby, D. J., Ferguson, M. W. J., Harrison, M. R. and Adzick, N. S. (1994) "Adult skin wounds in the fetal environment heal with scar formation," Ann. Surg.. 219 , $65-72$.

Lorenz, H. P. and Adzick, N. S. (1992) "Scarless wound repair in the fetus." Western J. Med., 159, 350-355.

Lorenz, H. P., Longaker, M. T., Perocha, L. A., Jennings, R. W., Harrison, M. R. and Adzick, N.S. (1992) "Scarless wound repair: A human fetal skin model," Development. 114, $253-259$.

Lorenz, H. P., Whitby, D. J., Longaker, M. T. and Adzick, N. S. (1993) "Fetal wound healing: The ontogeny of scar formation in the non-human primate," Ann. Surg., 217, 391-396.

Lorenz. H. P.. Lin, R. Y.. Longaker, M. T., Whitby, D. J. and Adzick, N. S. (1995) "The fetal fibroblast: The effector cell of scarless fetal skin repair," Plastic Reconstruct. Surg., 96, $1251-1261$.

Martin, P. and Lewis, J. (1992) "Actin cables and epidermal movement in embryonic wound healing," Nature, $\mathbf{3 6 0}$. $179-183$.

Martin, P., Dickson, M. C., Millan, F. A. and Akhurst, R. J (1993) Rapid induction and clearance of $\mathrm{TGF} \beta_{1}$ is an early response to wounding in the mouse embryo," Delelop. Genet., 14, $225-338$

Mast, B. A., Flood, L. C., Haynes, J. H., DePalma, R. L., Cohen, I. K., Dieglmann. R. F. and Krummel. T. M. (1991) "Hyaluronic acid is a major component of the matrix of fetal rabbit skin and wounds: Implications for healing by regeneration." Matrix, 11,63-68.

Mast. B. A., Dieglemann, R. F., Krummel, T. M. and Cohen. I. K. (1992) "Scarless wound healing in the mammalian fetus," Surg. Gynecol. Obste, 174, 441-451.

McCallion, R. L., Wood, J. and Ferguson, M. W. J. "Mannose6-phosphate reduces dermal scarring" Submitted. 
McCluskey, J., Hopkinson-Wooley, J., Luke, B. and Martin, P. (1993 "A study of wound healing in the E11.5 mouse embryo by light and electron microscopy," Tissues Cells, 25, 173-181.

Merkel, J. R., DiPaolo, B. R., Hallock, G. G. and Rice, D. C. (1988). "Types I and III collagen content of healing wounds in fetal and adult rats," Proc. Soc. Experimental Biol. Med. 187, 493-497.

Meuli, M., Lorenz, H. P., Hedrick, M. H., Sullivan, K. M., Harrison, M. R. and Adzick, N. S. (1995) "Scar formation in the fetal alimentary tract." J. Pediatric Surg., 30. 392-395.

Morykwas, M. J., Ditesheim, J. A., Ledbetter, M. S., Crook, E., White, W. L., Jennings, D. A. and Argeta, L. C. (1991) "Monbdelphis domesticus: A model for early developmental wound healing," Ann. Plastic Surg., 4, 327-331.

Pierce, G. F., Vandeberg, J., Rudolph, R., Tarpley, J. and Mustde, T. (1991) "Platelet derived growth factor-BB and transfbrming growth factor beta-1 selectively modulate glycosaminoglycans, collagen and myofibroblasts in excisional wounds," Amer. J. Path., 138, 629-646.

Purchio, A. F., Cooper, J. A., Brunner, A. M., Lioubin, M. N., Gentry, L. E., Kovacina, K. S., Roth, R. A. and Marquardt, H. (1988) "Identification of mannose-6-phosphate in two asparagine-linked sugar chains of recombinant TGF $\beta 1$ precursor," J. Biol. Chem., 263, 14211-14215.

Robinson, B. W. and Goss, A. N. (1981) "Intrauterine healing of fetal rat cheek wounds," Cleft Palate J., 18, 251-255.

Roswell; A. R. (1984) "The intrauterine healing of fetal muscle wounds: experimental study in the rat," Brit. J. Plastic Surg., $37,615-642$

Ruoslahti, E. and Yamaguchi, Y. (1991) "Proteoglycans as modulators of growth factor activities," Cell, 64, 867-869.

Schor, S. L., Schor, A. M., Rushton, G. and Smith, L. (1985) "Adult, fetal and transformed fibroblasts display different migratory phenotypes on collagen gels: Evidence for an isoform transition during fetal development," J. Cell Sci., 73, 221-\$34.

Schor, S. L., Schor, A. M., Grey, A. M., Chen, J., Rushton, G., Grant, M. and Ellis, I. (1989) "Mechanisms of action of migration stimulating factor (MSF) produced by fetal and cancer patient fibroblasts: Effect on hyaluronic acid synthesis," In vitro Cell Develop. Biol., 25, 737-746.

Scott, J. E. (1988) "Proteoglycan-fibrillar collagen interactions," Biochem. J., 252, 313-323.

Shah, M., Foreman, D. M. and Ferguson, M. W. J. (1992) "Control of scarring in adult wounds by neutralising antibody to TGF $\beta$," Lancet, 339, 213-214.
Shah, M., Foreman, D. M. and Ferguson, M. W. J. (1994) "Neutralising antibody to TGF $\beta 1,2$ reduces cutaneous scarring in adult rodents," J. Cell Sci., 107, 1137-1157.

Shah, M., Foreman, D. M. and Ferguson, M. W. J. (1995).

Shaw, L. M. and Olsen, B. R. (1991) "FACIT collagens: Diverse molecular bridges in extracellular matrices," Trends Biochem., 16. $191-194$.

Somasundaram, K. and Prathap, K. (1970) "Intra-uterine healing of skin wounds in rabbit fetuses," J. Pathol., 100, 81-86.

Somasundaram, K. and Prathap, K. (1972) "The effect of exclusion of amniotic fluid on intra-uterine healing of skin wounds in rabbit fetuses," J. Pathol., 107, 127-130.

Speranz, M. L., Valentini, G. and Calligaro, A. (1987) "Influence of fibronectin on fibrillogenesis of type I and type III collagen," Collagen Related Res., 7, 115-123.

Stern, M., Schmid, B., Dodson, T. B., Stern, R. and Kaban, K. B. (1992) "Fetal cleft lip repair in rabbits: Histology and role of hyaluronic acid," J. Oral Maxillofacial Surg., 22, 371-374.

Terrell, T. G., Working, P. K., Chow, C. P.. Green, C. P. and Green, J. D. (1993) "Pathology of recombinant human transforming growth factor- $\beta 1$ in rats and rabbits," Int. Rev. Experimental Pathol. 34, 43-67.

Turley, E. A., Austen, L., Vandeligt. K. and Clary, C. (1991) "Hyaluronan and a cell associated hyaluronan binding protein regulates the locomotion of ras-transformed cells," J. Cell Biol., 112, 104l-1047.

Van Vlasselaer, P., Borremans, B., van Gorp, U, Dasch, J. R. and DeWaal-Malefyt, R. (1994). "Interleukin-10 inhibits transforming growth factor- $\beta$ (TGF- $\beta$ ) synthesis required for osteo genic commitment of mouse bone marrow cells," J. Cell Biol., $124,569-577$.

Wahl, S. M, Hunt, D. A., Wakefield, N., McCartney-Francis, L. M., Wahl, A. B., Roberts, A. B. and Sporn, M. B. (1987) "Transforming growth factor beta (TGF- $\beta$ ) induces monocyte chemotaxis and growth factor production," Proc. Natl. Acad. Sci. USA, 84, 5788-5792.

Whitby, D. J. and Ferguson. M. W. J. (1991a) "The extracellular matrix of lip wounds in fetal, neonatal and adult mice." Development, 112, 651-668.

Whitby, D. J. and Ferguson, M. W. J. (1991b) "Immunolocalisation of growth factors in fetal wound healing," Develop. Biol., 147, 2207-2215.

Wider, T. M., Yager, J. S., Rittenberg, T., Hugo, N. E. and Erlich, P. (1993) "The inhibition of fibroblast populated collagen lattice contraction by human amniotic fluid: A chronologic examination," Plastic Reconstruct. Surg., 91, 1287-1293. 


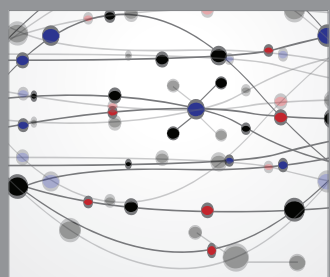

The Scientific World Journal
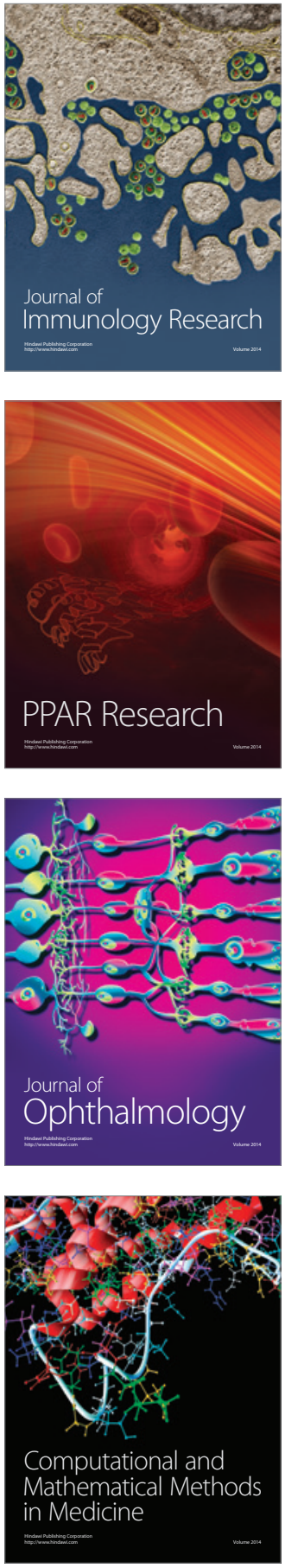

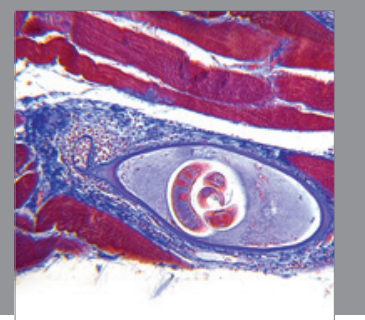

Gastroenterology

Research and Practice
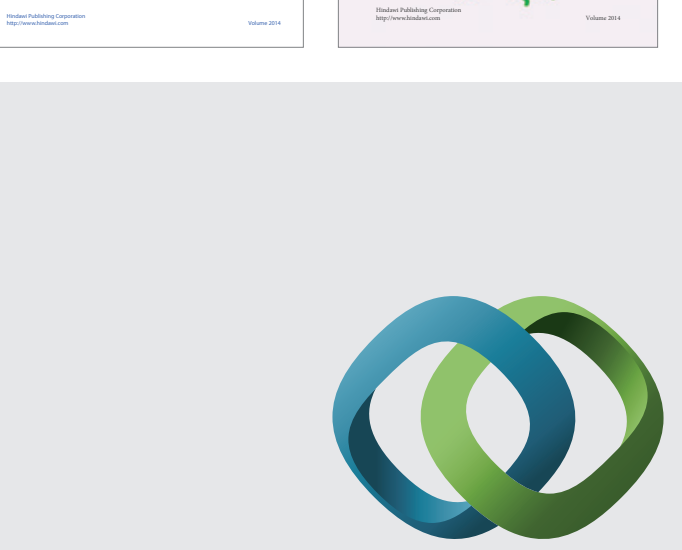

\section{Hindawi}

Submit your manuscripts at

http://www.hindawi.com
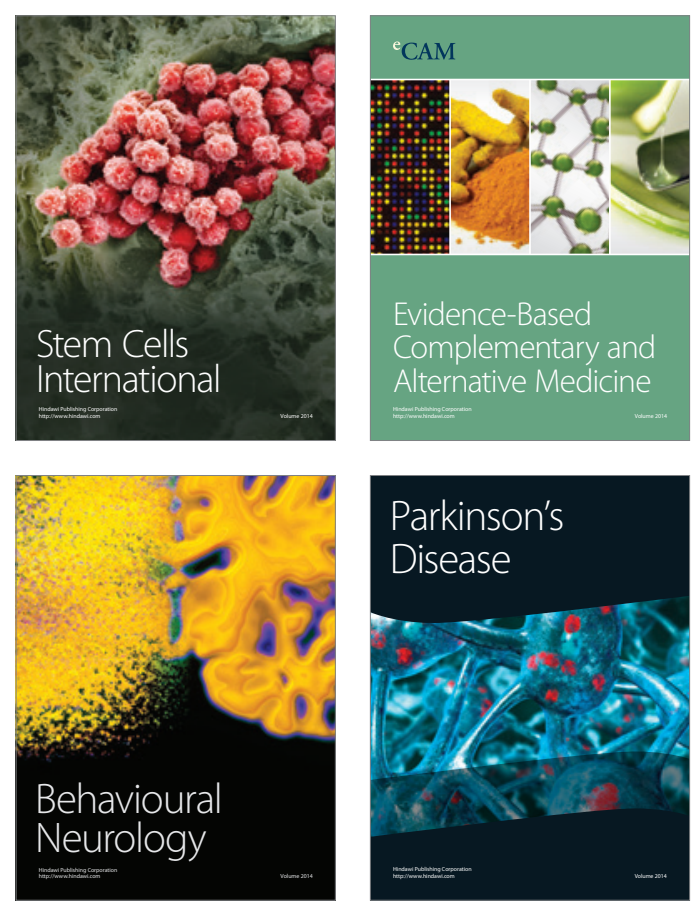

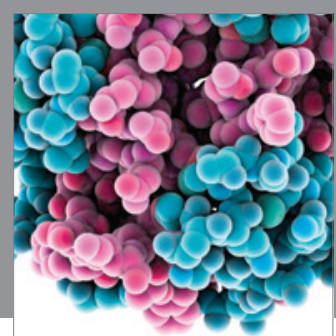

Journal of
Diabetes Research

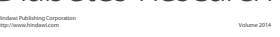

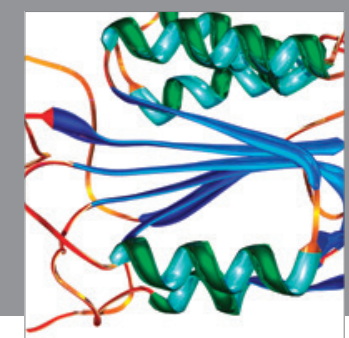

Disease Markers
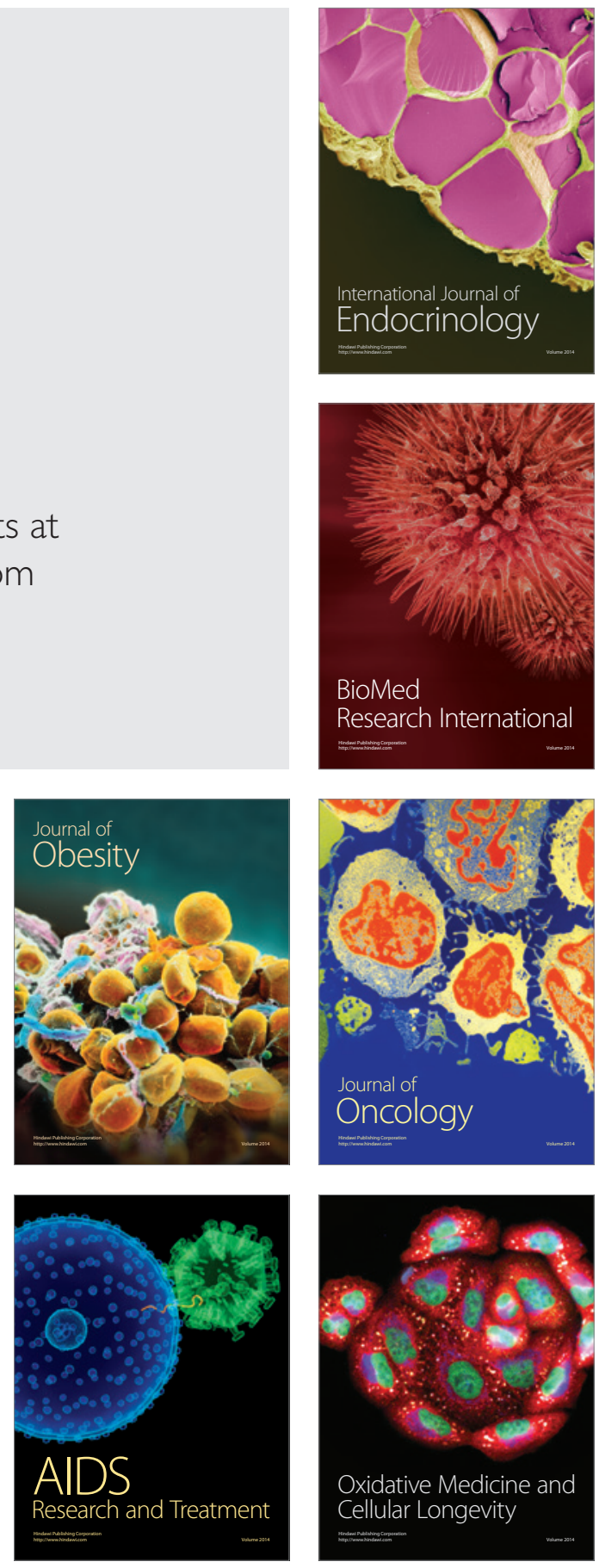